Check for updates

Cite this: Nanoscale Horiz., 2020, 5,1447

Received 30th July 2020,

Accepted 18th September 2020

DOI: $10.1039 / \mathrm{d} 0 \mathrm{nh} 00465 \mathrm{k}$

rsc.li/nanoscale-horizons

\section{Are two-dimensional materials radiation tolerant?}

\author{
Arkady V. Krasheninnikov (D) *ab
}

Two-dimensional (2D) materials have many unique properties, which can be exploited in various applications. In particular, electronic devices based on 2D materials should ideally be suited for the operation in outer cosmic space due to their low weight, small size and low power consumption. This brings about the issue of their radiation hardness, or tolerance, which has recently been addressed in a number of studies. The results of these investigations are somewhat counterintuitive: although one can naively expect that atomically thin structures should easily be destroyed by the beams of energetic particles, the devices made from 2D materials were reported to exhibit extraordinary radiation hardness. In this Focus article, an overview of the recent studies on the subject is given, followed by the discussion of the origin of the reported high tolerance, which is inherently related to the response of 2D materials, the systems with the reduced dimensionality, to irradiation. The analysis of the experimental and theoretical data on the behavior of $2 \mathrm{D}$ systems under irradiation indicates that although free-standing 2D materials can indeed be referred to as radiation resilient systems under irradiation conditions corresponding to the outer space, this is generally not the case, as the environment, e.g., the substrate, can strongly influence the radiation tolerance of $2 \mathrm{D}$ materials and devices based on these systems.

\section{Introduction}

The isolation of a single sheet of graphene ${ }^{1}$ has directed the attention of the scientific community towards two-dimensional (2D) materials. Many 2D systems beyond graphene, e.g., hexagonal boron nitride (h-BN), transition metal dichalcogenides (TMDs), and metal-organic framework nanosheets have been produced by mechanical $^{2}$ and chemical $^{3}$ exfoliation of their layered bulk counterparts, as well as by growth techniques based on chemical catalyst-free vapor-solid methods, ${ }^{4}$ atomic layer deposition, ${ }^{5}$ or liquid/air interfacial synthesis techniques. ${ }^{6}$ 2D materials have been demonstrated to have many unique properties, which can be exploited in various applications, see, e.g., ref. 7 for an overview. In particular, electronic devices based on 2D materials, such as transistors, sensors, single-photon emitters, etc. should ideally be suited for aerospace and open space applications due to their low weights, small sizes and low power consumption. This means that they will be operating in a radiation-hostile environment, as the devices will be subjected to fluxes of radiation, such as high-energy protons and heavier ions, electrons, X-ray and $\gamma$-rays. This brings about the issue of their radiation tolerance, which can also be referred to as radiation hardness or susceptibility.

\footnotetext{
${ }^{a}$ Institute of Ion Beam Physics and Materials Research, Helmholtz-Zentrum Dresden-Rossendorf, 01314 Dresden, Germany. E-mail: a.krasheninnikov@hzdr.de

${ }^{b}$ Department of Applied Physics, Aalto University School of Science,

P.O. Box 11100, 00076 Aalto, Finland
}

Radiation tolerance is a characteristic of a material subjected to beams of energetic particles or $\gamma$-rays, which indicates how long a device based on this material can operate under constant flux of energetic particles in a radiation-hostile environment. $^{8}$ The malfunction of the device is normally associated with the accumulation of irradiation-induced defects, but other mechanisms of device failure are possible, e.g., in addition to lattice displacements by direct particle knock-on or ionization damage, high currents related to the generation of electron-hole pairs can destroy the device. A quantitative analysis of materials in the context of radiation tolerance is difficult, as the criterion of the failure obviously depends on the device. Nevertheless one can speak of the concentration of defects produced in the sample after exposure to a certain fluence of energetic particles. It should be pointed out, though, that even for the same concentration of defects, their effects on the electronic properties can be different and depend on where the defect-induced electronic states appear (in the gap or in the valence/conduction band) upon defect formation, as discussed, e.g., for 2D TMDs. ${ }^{9}$ For systems irradiated with different particles one can also estimate the amount of absorbed energy per mass unit of the material, but for $2 \mathrm{D}$ system this quantity is not well defined, as discussed below.

Radiation hardness of 2D materials has recently been addressed in a number of studies. ${ }^{10-13}$ The results of these investigations were somewhat puzzling: although one can expect that atomically thin structures should easily be destroyed by the beams of energetic particles, the devices made from 2D materials 
were reported to exhibit rather high radiation tolerance in terms of the irradiation fluence. Even a conclusion about their 'extraordinary' hardness (with respect to the bulk counterparts) was made after the estimates of the life time of the devices based on TMDs, which corresponded to at least hundreds of years of unshielded exposure to radiation in open space. ${ }^{13}$

In this article, an overview of the recent studies on the subject is given with the main focus on ion irradiation, followed by the discussion of the origin of the reported high tolerance, which is inherently related to the response of $2 \mathrm{D}$ materials to particle irradiation. We also address the effects of the environment on the defect production in 2D systems, which naturally affects their radiation hardness.

\section{Experiments on irradiation tolerance of $2 \mathrm{D}$ materials}

The response of $2 \mathrm{D}$ materials to irradiation (mostly with ions and electrons) has extensively been studied, see ref. 14-18 for an overview. In particular, the effects of particle irradiation on the electronic transport properties have been addressed. ${ }^{19-23}$ However, normally rather low ion energies were used in these studies (e.g., $30 \mathrm{keV}$ He ions), and in the following we concentrate on the data relevant to the outer space or other radiation-hostile environments (particle accelerators, nuclear facilities, etc.)

The evolution of the electrical characteristics of a $\mathrm{MoS}_{2}$ based field-effect transistor (FET) under irradiation with $10 \mathrm{MeV}$ proton beams has been studied by Kim et al. ${ }^{12}$ For a low proton fluence of $10^{12} \mathrm{~cm}^{-2}$, the device performance remained almost the same as before the sample was subjected to irradiation. For a higher proton fluence of $10^{13}$ or $10^{14} \mathrm{~cm}^{-2}$, a dramatic reduction in the conductance of the devices was observed, as evident form Fig. 1, which shows the images of the sample, irradiation setup and drain-source current-to voltage characteristics before and after the exposure to the beam. The deterioration of the device performance was associated not with the defects produced in the $2 \mathrm{D}$ system, but attributed to the presence of charge traps in the oxide substrate and their subsequent impact on the electrical characteristics of the device.

Ochedowski et $a{ }^{10}{ }^{10}$ have investigated the performance of field-effect transistors based on graphene and $\mathrm{MoS}_{2}$ under $1.14 \mathrm{GeV} \mathrm{U}^{28+}$ ion beam. The highest applied fluence of $4 \times 10^{11}$ ions per $\mathrm{cm}^{2}$ was found to destroy the $\mathrm{MoS}_{2}$-based transistor, while the device with graphene could operate, although its performance deteriorated considerably. It is interesting to note that the improvements in the operation of some devices was reported, which may have the same origin as defect annealing in graphene upon low-fluence high energy ion irradiation ${ }^{24}$ or the enhancement of the electronic properties of $\mathrm{MoS}_{2}$ after lowfluence He ion bombardment. ${ }^{19}$

The behavior of electronic devices such as field-effect transistors based on 2D MoS 2 and $\mathrm{WS}_{2}$ and single-photon sources based on h-BN under combined $\gamma$-ray, proton and electron beam irradiation has also been studied ${ }^{11}$ in the context of their radiation tolerance for space applications. It was found that the devices showed negligible changes in performance after the irradiation with the doses equivalent to what one can expect after being for $10^{3}$ years at $500 \mathrm{~km}$ above the polar caps. Counter-intuitively, monolayer $\mathrm{WS}_{2}$ exhibited decreased defect densities under high-dose $\gamma$-radiation, as identified by an increase in photo-luminescence, carrier lifetime and a change in doping ratio proportional to the photon flux. The underlying mechanism was assumed to be related to radiationinduced defect healing, possibly due to the passivation of sulfur vacancies by the dissociated oxygen, in line with the observations reported by Ochedowski et al. ${ }^{10}$

The assessment of the radiation hardness of $2 \mathrm{D}$ materials using typical electrical measurements is not straightforward due to the fact that the substrate can trap charges. Thus, to further investigate how the defects produced in the substrate affect the
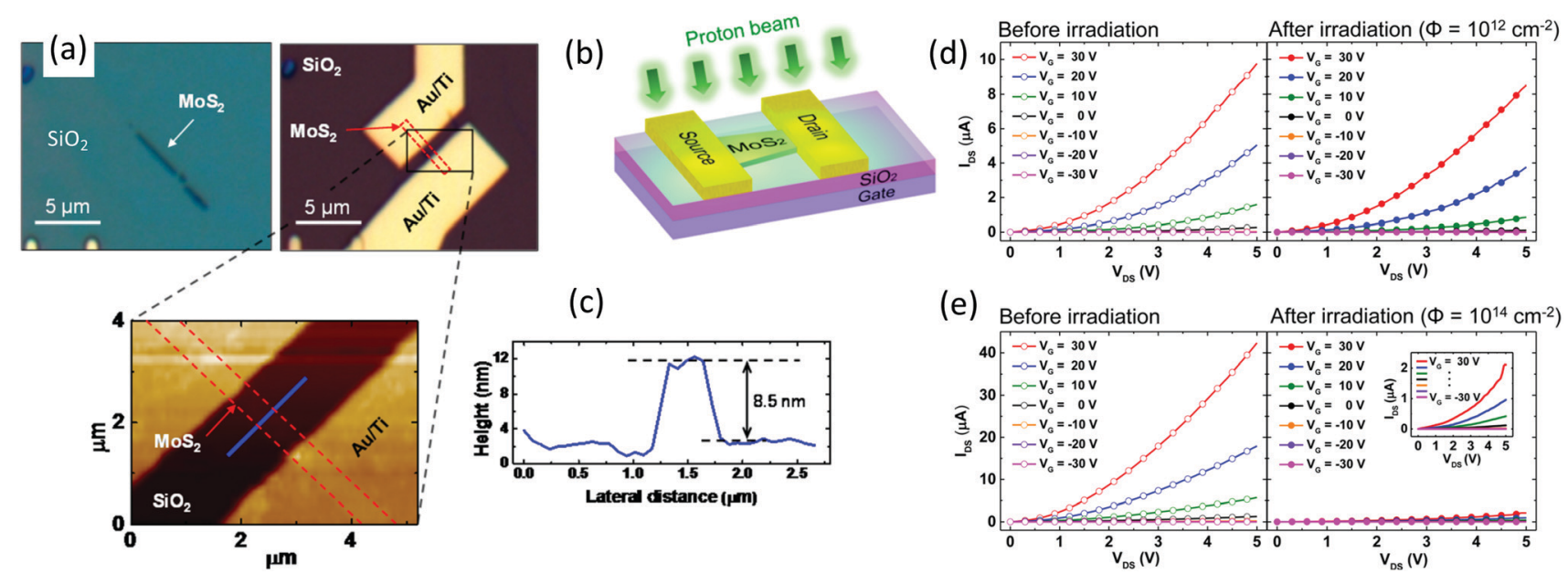

(e)

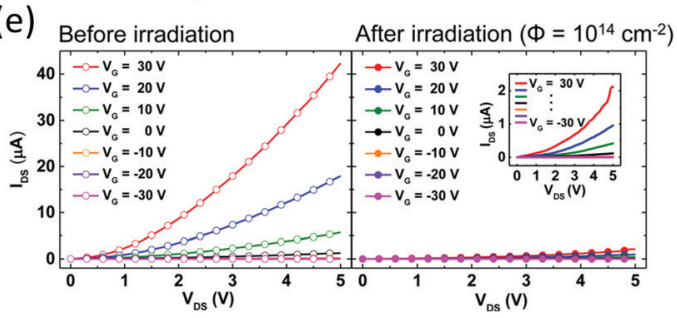

Fig. 1 (a) Optical images showing a MoS 2 FET device. (b) Schematic illustration of proton beam irradiation of the device. (c) AFM image of the MoS 2 FET device with a cross-sectional topographic profile indicated by the blue line. Red dashed lines in (a and c) indicate the flakes. (d) Representative electrical characteristics of $\mathrm{MoS}_{2}$ FET devices before and after proton irradiation with a beam fluence of $10^{12} \mathrm{~cm}^{-2}$. The same for a fluence of $10^{14} \mathrm{~cm}{ }^{-2}$. The images adapted with permission from ref. 12 
performance of the irradiated FET devices based on a 2D material, in a recent study by Arnold et al., ${ }^{13}$ a special experimental setup was used to decouple the radiation impact due to the defects in the $2 \mathrm{D}$ semiconducting channel and the irradiation-induced change in the oxide dielectric. Specifically, the substrate and semiconducting 2D material $\left(\mathrm{MoS}_{2}\right)$ were irradiated separately, then the device was made (irradiated $\mathrm{MoS}_{2}$ plus pristine substrate and vice versa), and its transport characteristics measured and compared to those obtained from the device which was assembled from the pristine components and then irradiated. The results showed that the interface states play a major role in the electrical characteristic following irradiation with a He ion fluence of $10^{15}$ ions per $\mathrm{cm}^{2}$, whereas oxide charges have dominant effect for the case exposed to a proton fluence of $1.26 \times 10^{16}$ ions per $\mathrm{cm}^{2}$. A conclusion about "extraordinary" radiation hardness of atomically thin $\mathrm{MoS}_{2}$ was made.

\section{Response of 2D materials to energetic particle irradiation}

To rationalize the results of the experiments discussed above, it is necessary to address first the defect production in $2 \mathrm{D}$ materials under energetic particle irradiation. Here we concentrate on ion bombardment, and analyze the general trends in damage creation, accumulation and annealing. We do not explicitly present the results of numerous experimental and theoretical studies, and refer the reader to the recent comprehensive review articles ${ }^{14-18,25}$ on the subject. The studies indicate that although the impacts of energetic ions give rise to defects in both bulk and 2D materials, the response of $2 \mathrm{D}$ materials to irradiation can be quite different from that in bulk systems, as schematically illustrated in Fig. 2 .

Defects can appear through nuclear collisions or, at higher ion energis through electronic excitations and ionization with the threshold values for defect production being dependent on ion and target atom masses, ion energy, ion charge state and the electronic structure of the target. In both bulk and $2 \mathrm{D}$ systems, at low ion energies (below $100 \mathrm{eV}$ ) the defects are formed through ballistic displacement of atoms when ion energy exceeds a certain threshold value $E_{\mathrm{th}}^{\mathrm{n}} \cdot{ }^{27-31}$ In supported $2 \mathrm{D}$ materials, ${ }^{19,32-35}$ that is deposited on a substrate, $E_{\mathrm{th}}^{\mathrm{n}}$ is expected to be higher, as the substrate should stop the displaced atoms and facilitate the immediate annihilation of vacancies. At higher ion energies, the atoms sputtered from the substrate or backscattered ions can create additional damage in the $2 \mathrm{D}$ target. In fact, for light ions with medium
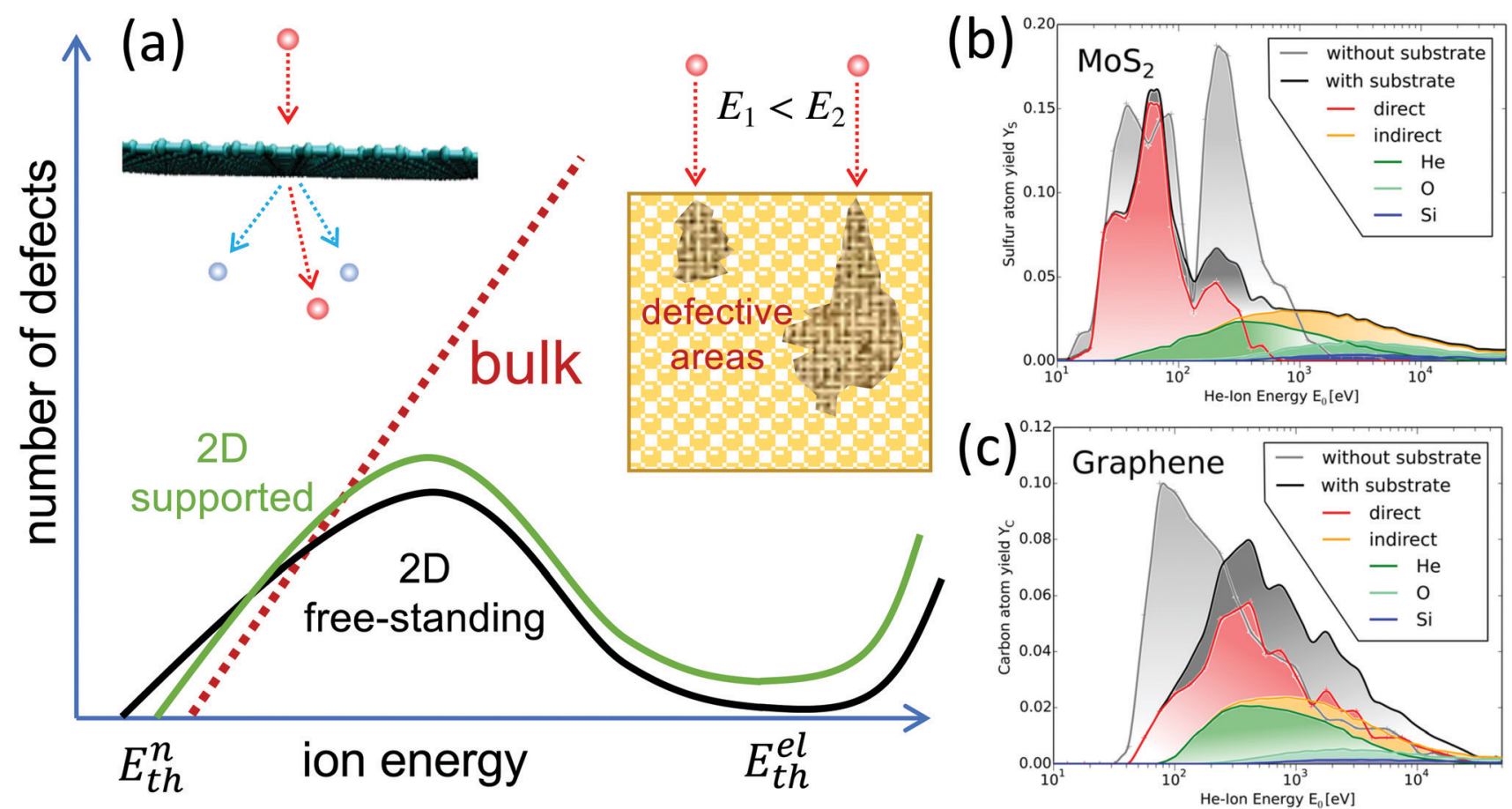

Fig. 2 (a) Schematic illustration of the main differences in defect production in bulk and free-standing 2D materials under impacts of energetic ions. In both systems, at small ion energies defects appear through ballistic displacement of atoms when ion energy exceeds a certain threshold value $E_{\text {th. }}^{n}$ In supported 2D materials $E_{\mathrm{th}}^{\mathrm{n}}$ is higher, as the substrate stops the displaced atoms. At higher ion energies, the atoms sputtered from the substrate or backscattered ions can create additional damage in the 2D target. In both $2 \mathrm{D}$ and bulk materials the number of defects first grows up with ion energy. However, defect production decreases at some point in 2D systems as less ion energy is deposited into the sample due to a decrease in cross-section and the absence of collisional cascades. In contrast, all ion energy is eventually transferred to defects and heat in the bulk system, and more defects are produced, although deeper in the sample. When energy deposited in electronic excitations exceeds also the threshold value $E_{\mathrm{th}}^{\mathrm{el}}$, the number of defects in the 2D system can start growing again. (b and c) Examples of damage productions in 2D systems as obtained in atomistic simulations of He ion irradiation. Average number of atoms sputtered from $\mathrm{MoS}_{2}$ (b) and graphene (c) per He ion impact as functions of ion energy. The corresponding numbers for freestanding monolayers (grey) and the direct sputtering (red) are shown for comparison. The images adapted with permission from ref. 26. 
energies, the defect production in 2D systems can be completely governed by the backscattered ions and atoms sputtered from the substrate rather than by the direct ion impacts. ${ }^{26}$ In both $2 \mathrm{D}$ and bulk materials, the number of defects per ion impact first grows up with ion energy. However, it starts decreasing at some point in 2D systems as less ion energy is deposited into the sample due to a drop in the cross-section for atom displacement and the absence of collisional cascades, as established within the framework of the binary collision approximation. ${ }^{18,36}$ The maximum in defect production is in the range of $10^{2}-10^{3} \mathrm{eV}$. In contrast, all ion energy is eventually transferred to defects and heat in the bulk system (assuming that the ion range is smaller than the thickness of the target), and more defects are produced with increasing energy, although deeper in the sample. The collisional cascades do not develop in 2D materials especially for the free-standing sheets (deposited on a grid or suspended over a trench in the substrate), and most of the energy transferred from the ion to the primary recoil atom is taken away from the system. At higher energies ions go through the 2D system without producing much damage through ballistic collisions.

Defects start appearing again at higher ion energies due to ionization effects, that is a large amount of energy deposited into electronic excitations followed by the conversion of the excitation energy into defects. The threshold $E_{\mathrm{th}}^{\mathrm{el}}$ for defect production through this channel depends on the electronic structure of the material, and can be very high for $2 \mathrm{D}$ systems with high electron mobility and thermal conductance: for example, the experiments and simulations ${ }^{37-39}$ indicate that holes in graphene appear at ion energies in tens of $\mathrm{MeV}$ range corresponding to electronic stopping power of a few keV per graphene layer. To this end, conducting $2 \mathrm{D}$ materials should be more radiation-tolerant than insulating.

The substrate, however, decreases the threshold. ${ }^{39}$ The defects in the supported graphene appear due to the atoms sputtered from the core region of the track formed in the substrate. Moreover, experiments ${ }^{40}$ showed that graphene on a dielectric substrate can be cut and folded under impacts of swift heavy ions (with energies of the order of $100 \mathrm{MeV}$ ) with grazing trajectories due to a combination of defect creation in the graphene layer and hillock creation in the substrate. The folded parts were reported to be up to hundreds of $\mathrm{nm}$ in length, indicating that the radiation hardness of supported graphene is in general questionable, as impacts of ions with grazing trajectories cannot be excluded.

The annealing of defects is also important in the context of the radiation hardness of $2 \mathrm{D}$ materials. Contrary to the bulk systems, where the effects of the environment on defect evolution are normally ignored, 2D materials consist of essentially surface only, so that additional defects can appear (e.g., due to the interaction with the reactive species like oxygen molecules) or the other way around, disappear - an example is the self-healing of vacancies in graphene due to the dissociation of hydrocarbon molecules. ${ }^{41}$ On the other hand, while impacts of energetic ions produce interstitial-vacancy pairs in bulk systems, only vacancies will be left in the 2D structure, as displaced atoms will be sputtered away.
This also complicates the assessment of the deposited energy per mass unit of the system. For supported 2D systems, the evolution of defects, e.g., vacancies, may also be dominated by the interaction of defects with the substrate. ${ }^{42-44}$

\section{Conclusions and outlook}

As evident from the above, putting aside the limit of low (tens of $\mathrm{eV}$ ) ions, projectiles with the same energy will produce less defects in free-standing 2D structures than bulk materials. This is related to several factors: (i) absense of collisional cascades, (ii) the recoil atoms are predominantly sputtered away, and as they may have considerable kinetic energy, the actual deposited energy can be much smaller than the energy lost by the projectile. (iii) In situ healing of defects during the irradiation due to the interaction with the environment provided that the species required to 'mend' the damage are present. Thus, using the number of defects produced in the system after the ion impact as a measure, 2D systems can indeed be regarded as radiation-tolerant systems.

Conducting 2D materials should be more radiation-tolerant than insulating under impact of high-energy particles, when ionization and electron excitations are the dominant mechanisms of damage creation. Presumably, radiation hardness can be enhanced by doping the system, provided that it is consistent with the device operation. Besides, making heterostructures by combining semiconducting/insulating 2D materials with metallic ones can increase the stability of the former under high-energy ion bombardment by decreasing charge accumulation and also suppressing sputtering, similar to the results of the experiments on the behavior of TMD/graphene heterostructures under electron beam. ${ }^{45}$ The results of very recent experiments ${ }^{46}$ on the response of such heterostructures to impacts of highly-charged ions seem to confirm this.

However, in the devices where supported 2D materials will be used (and this is likely going to be the most common case), the substrate can strongly influence the behavior of 2D materials under irradiation, and even a single impact of a high-energy ion can completely destroy the $2 \mathrm{D}$ component due to atoms sputtered from the substrate or the development of mechanical strain in the $2 \mathrm{D}$ system due to deformation (e.g., hillock creation) in the substrate.

Overall, more experimental work is required to systematically study the response of various devices based on $2 \mathrm{D}$ materials to irradiation in the context of their radiation hardness. The recently suggested approach ${ }^{13}$ where substrate and 2D system are irradiated separately, then the devices are made, and their characteristics are compared to that of the devices assembled from pristine components and then irradiated, appears to be the most promising route to separate the effects of the environment and 'intrinsic' radiation hardness of a $2 \mathrm{D}$ material. The 'traditional' devices should also be studied under exactly the same conditions if a direct comparison is required.

\section{Conflicts of interest}

There are no conflicts to declare. 


\section{Acknowledgements}

I would like to thank S. Facsko, G. Hlawacek, M. Ghorbani-Asl, and S. Kretschmer for discussions. I also acknowledge DFG for support within the projects KR 4866/2-1 (406129719).

\section{Notes and references}

1 K. S. Novoselov, A. K. Geim, S. V. Morozov, D. Jiang, Y. Zhang, S. V. Dubonos, I. V. Grigorieva and A. A. Firsov, Science, 2004, 306, 666-669.

2 B. Radisavljevic, A. Radenovic, J. Brivio, V. Giacometti and A. Kis, Nat. Nanotechnol., 2011, 6, 147-150.

3 J. N. Coleman, M. Lotya, A. O’Neill, S. D. Bergin, P. J. King, U. Khan, K. Young, A. Gaucher, S. De, R. J. Smith, I. V. Shvets, S. K. Arora, G. Stanton, H.-Y. Kim, K. Lee, G. T. Kim, G. S. Duesberg, T. Hallam, J. J. Boland, J. J. Wang, J. F. Donegan, J. C. Grunlan, G. Moriarty, A. Shmeliov, R. J. Nicholls, J. M. Perkins, E. M. Grieveson, K. Theuwissen, D. W. McComb, P. D. Nellist and V. Nicolosi, Science, 2011, 331, 568-571.

4 R. Zhang, Z. D. Zhang, Z. S. Wang, S. Wang, W. Wang, D. J. Fu and J. Liu, Appl. Phys. Lett., 2012, 101, 011905.

5 J. Cai, X. Han, X. Wang and X. Meng, Matter, 2020, 2, 587-630. 6 M. Zhao, Q. Lu, Q. Ma and H. Zhang, Small Methods, 2017, 1, 1600030.

7 A. C. Ferrari, F. Bonaccorso, V. Fal'ko, K. S. Novoselov, S. Roche, P. Bøggild, S. Borini, F. H. L. Koppens, V. Palermo, N. Pugno, J. A. Garrido, R. Sordan, A. Bianco, L. Ballerini, M. Prato, E. Lidorikis, J. Kivioja, C. Marinelli, T. Ryhänen, A. Morpurgo, J. N. Coleman, V. Nicolosi, L. Colombo, A. Fert, M. Garcia-Hernandez, A. Bachtold, G. F. Schneider, F. Guinea, C. Dekker, M. Barbone, Z. Sun, C. Galiotis, A. N. Grigorenko, G. Konstantatos, A. Kis, M. Katsnelson, L. Vandersypen, A. Loiseau, V. Morandi, D. Neumaier, E. Treossi, V. Pellegrini, M. Polini, A. Tredicucci, G. M. Williams, B. Hee Hong, J.-H. Ahn, J. Min Kim, H. Zirath, B. J. van Wees, H. van der Zant, L. Occhipinti, A. Di Matteo, I. A. Kinloch, T. Seyller, E. Quesnel, X. Feng, K. Teo, N. Rupesinghe, P. Hakonen, S. R. T. Neil, Q. Tannock, T. Löfwander and J. Kinaret, Nanoscale, 2015, 7, 4598-4810.

8 H. Spieler, AIP Conf. Proc., 1997, 23-49.

9 M. Pandey, F. A. Rasmussen, K. Kuhar, T. Olsen, K. W. Jacobsen and K. S. Thygesen, Nano Lett., 2016, 16, 2234-2239.

10 O. Ochedowski, K. Marinov, G. Wilbs, G. Keller, N. Scheuschner, D. Severin, M. Bender, J. Maultzsch, F. J. Tegude and M. Schleberger, J. Appl. Phys., 2013, 113, 214306.

11 T. Vogl, K. Sripathy, A. Sharma, P. Reddy, J. Sullivan, J. R. Machacek, L. Zhang, F. Karouta, B. C. Buchler, M. W. Doherty, Y. Lu and P. K. Lam, Nat. Commun., 2019, 10, 1202.

12 T.-Y. Kim, K. Cho, W. Park, J. Park, Y. Song, S. Hong, W.-K. Hong and T. Lee, ACS Nano, 2014, 8, 2774-2781.

13 A. J. Arnold, T. Shi, I. Jovanovic and S. Das, ACS Appl. Mater. Interfaces, 2019, 11, 8391-8399.
14 Z. Li and F. Chen, Appl. Phys. Rev., 2017, 4, 011103.

15 M. Schleberger and J. Kotakoski, Materials, 2018, 11, 1885.

16 G.-Y. Zhao, H. Deng, N. Tyree, M. Guy, A. Lisfi, Q. Peng, J.-A. Yan, C. Wang and Y. Lan, Appl. Sci., 2019, 9, 678.

17 R. C. Walker, T. Shi, E. C. Silva, I. Jovanovic and J. A. Robinson, Phys. Status Solidi A, 2016, 213, 3065-3077.

18 A. V. Krasheninnikov and K. Nordlund, J. Appl. Phys., 2010, 107, 071301.

19 D. S. Fox, Y. Zhou, P. Maguire, A. O’Neill, C. Ó’Coileáin, R. Gatensby, A. M. Glushenkov, T. Tao, G. S. Duesberg, I. V. Shvets, M. Abid, M. Abid, H.-C. Wu, Y. Chen, J. N. Coleman, J. F. Donegan and H. Zhang, Nano Lett., 2015, 15, 5307-5313.

20 M. G. Stanford, P. R. Pudasaini, A. Belianinov, N. Cross, J. H. Noh, M. R. Koehler, D. G. Mandrus, G. Duscher, A. J. Rondinone, I. N. Ivanov, T. Z. Ward and P. D. Rack, Sci. Rep., 2016, 6, 27276.

21 L. Tapasztó, G. Dobrik, P. Nemes-Incze, G. Vertesy, P. Lambin and L. P. Biró, Phys. Rev. B: Condens. Matter Mater. Phys., 2008, 78, 233407.

22 S. Nakaharai, T. Iijima, S. Ogawa, S. L. Li, K. Tsukagoshi, S. Sato and N. Yokoyama, Phys. Status Solidi C, 2013, 10, 1608-1611.

23 Y. Liu, Z. Gao, Y. Tan and F. Chen, ACS Nano, 2018, 12, 10529-10536.

24 S. Kumar, A. Tripathi, F. Singh, S. A. Khan, V. Baranwal and D. K. Avasthi, Nanoscale Res. Lett., 2014, 9, 126.

25 A. V. Krasheninnikov and F. Banhart, Nat. Mater., 2007, 6, 723-733.

26 S. Kretschmer, M. Maslov, S. Ghaderzadeh, M. GhorbaniAsl, G. Hlawacek and A. V. Krasheninnikov, ACS Appl. Mater. Interfaces, 2018, 10, 30827-30836.

27 O. Lehtinen, J. Kotakoski, A. V. Krasheninnikov, A. Tolvanen, K. Nordlund and J. Keinonen, Phys. Rev. B: Condens. Matter Mater. Phys., 2010, 81, 153401.

28 E. P. Bellido and J. M. Seminario, J. Phys. Chem. C, 2012, 116, 4044.

29 S. Zhao and J. Xue, Phys. Rev. B: Condens. Matter Mater. Phys., 2012, 86, 165428.

30 K. Yoon, A. Rahnamoun, J. L. Swett, V. Iberi, D. A. Cullen, I. V. Vlassiouk, A. Belianinov, S. Jesse, X. Sang, O. S. Ovchinnikova, A. J. Rondinone, R. R. Unocic and A. C. Van Duin, ACS Nano, 2016, 10, 8376-8384.

31 W. Li, L. Liang, S. Zhao, S. Zhang and J. Xue, J. Appl. Phys., 2013, 114, 234304.

32 W. Li, X. Wang, X. Zhang, S. Zhao, H. Duan and J. Xue, Sci. Rep., 2015, 5, 9935.

33 M. Kalbac, O. Lehtinen, A. V. Krasheninnikov and J. Keinonen, Adv. Mater., 2013, 25, 1004-1009.

34 G. Buchowicz, P. R. Stone, J. T. Robinson, C. D. Cress, J. W. Beeman and O. D. Dubon, Appl. Phys. Lett., 2011, 98, 032102.

35 Q. Ma, P. M. Odenthal, J. Mann, D. Le, C. S. Wang, Y. Zhu, T. Chen, D. Sun, K. Yamaguchi, T. Tran, M. Wurch, J. L. McKinley, J. Wyrick, K. Magnone, H. Bartels Tony, T. S. Rahman, R. Kawakami and L. Bartels, J. Phys.: Condens. Matter, 2013, 25, 252201. 
36 M. Nastasi, J. Mayer and J. K. Hirvonen, Ion-Solid Interactions: Fundamentals and Applications, Cambridge University Press, Cambridge, 1996.

37 H. Vazquez, E. Ahlgren, O. Ochedowski, A. Leino, R. Mirzayev, R. Kozubek, H. Lebius, M. Karlušic, M. Jakšic, A. Krasheninnikov, J. Kotakoski, M. Schleberger, K. Nordlund and F. Djurabekova, Carbon, 2017, 114, 511-518.

38 N. A. Nebogatikova, I. V. Antonova, S. V. Erohin, D. G. Kvashnin, A. Olejniczak, V. A. Volodin, A. V. Skuratov, A. V. Krasheninnikov, P. B. Sorokin and L. A. Chernozatonskii, Nanoscale, 2018, 10, 14499-14509.

39 S. Zhao and J. Xue, Carbon, 2015, 93, 169-179.

40 S. Akcöltekin, H. Bukowska, T. Peters, O. Osmani, I. Monnet, I. Alzaher, B. B. D'Etat, H. Lebius and M. Schleberger, Appl. Phys. Lett., 2011, 98, 103103.
41 R. Zan, Q. Ramasse, U. Bangert and K. S. Novoselov, Nano Lett. , 2012, 12, 3936-3940.

42 S. Standop, O. Lehtinen, C. Herbig, G. Lewes-Malandrakis, F. Craes, J. Kotakoski, T. Michely, A. V. Krasheninnikov and C. Busse, Nano Lett., 2013, 13, 1948-1955.

43 C. L. Wu, H. T. Lin, H. A. Chen, S. Y. Lin, M. H. Shih and C. W. Pao, Mater. Today Commun., 2018, 17, 60-68.

44 H. Cun, M. Iannuzzi, A. Hemmi, J. Osterwalder and T. Greber, Surf. Sci., 2015, 634, 95-102.

45 T. Lehnert, O. Lehtinen, G. Algara-Siller and U. Kaiser, Appl. Phys. Lett., 2017, 110, 033106.

46 J. Schwestka, H. Inani, M. Tripathi, A. Niggas, N. McEvoy, F. Libisch, F. Aumayr, J. Kotakoski and R. A. Wilhelm, ACS Nano, 2020, 14, 10536-10543. 